\title{
Karyological Analysis and Nature Polymorphics of Nucleolar Organizer Regions in the Dusky Leaf Monkey, Trachypithecus obscurus (Primate, Colobinae), by Conventional, GTG-, CBG-Banding, and High-Resolution Techniques
}

\author{
Alongklod Tanomtong ${ }^{1 *}$, Sumpars Khunsook ${ }^{1}$, Weeranuch Wonkaonoi ${ }^{1}$, \\ Wiwat Sangpakdee ${ }^{2}$, Roungvit Bunjongrat ${ }^{3}$ and La-orsri Sanoamuang ${ }^{1}$ \\ ${ }^{1}$ Applied Taxonomic Research Center (ATRC), Department of Biology, \\ Faculty of Science, Khon Kaen University, Khon Kaen 40002, Thailand \\ ${ }^{2}$ Biology Program, Faculty of Science, Udon-Thani Rajabhat University, \\ Muang, Udon-Thani 41000, Thailand \\ ${ }^{3}$ Major Genetics, Department of Botany, Faculty of Science, Chulalongkorn University, \\ Paya-Thai, Bangkok 10300, Thailand
}

Received April 6, 2011; accepted April 29, 2011

\begin{abstract}
Summary A discovery of polymorphic nucleolar organizer regions (NORs) and karyological analysis in the dusky leaf monkey (Trachypithecus obscurus) from Thailand were studied. Blood samples were taken from 2 male and 1 female leaf monkeys. After standard whole blood lymphocytes had been cultured at $37^{\circ} \mathrm{C}$ for $96 \mathrm{~h}$ in presence of colchicine, metaphase spreads were performed on microscopic slides and air-dried. Conventional, GBG-, CBG-banding and high-resolution techniques were applied to stain the chromosomes. The results showed that the diploid chromosome number of T. obscurus was $2 n=44$ and the fundamental number (NF) for both sexes was 88 . The types of autosomes were 6 large metacentric, 12 large submetacentric, 2 large acrocentric, 4 medium metacentric, 10 medium submetacentric, 2 small metacentric, 4 small submetacentric, and 2 small acrocentric chromosomes. The $\mathrm{X}$ chromosome was a large submetacentric chromosome and the $\mathrm{Y}$ chromosome was a small submetacentric chromosome. In addition, the long arm near centromere of chromosome pair 17 showed clearly observable NORs. This is the first report on nature polymorphism in NORs in T. obscurus. The results showed that a heteromorphism of 1 female had different size of NORs for chromosome pair $17 \mathrm{~s}$, while 2 males had an equal size of both chromosome pair $17 \mathrm{~s}$ with a homomorphism. From the GTG-banding and high-resolution techniques, the numbers of bands and locations in the T. obscurus were 235 and 314, respectively, and each chromosome pair could be clearly differentiated. CBG-banding showed C-positive (dark bands) on the centromeres of all autosomes but showed C-negative (light bands) on the Y chromosome. Moreover, we also found interstitial dark bands on the telomeres of long arms which result from centric fusion or tandem fusion. The karyotype formula for T. obscurus could be deduced as: $2 n$ (diploid) $44=\mathrm{L}_{6}^{\mathrm{m}}+\mathrm{L}_{12}^{\mathrm{sm}}+\mathrm{L}_{2}^{\mathrm{a}}+\mathrm{M}_{4}^{\mathrm{m}}+\mathrm{M}_{10}^{\mathrm{sm}}+\mathrm{S}_{2}^{\mathrm{m}}+$ $\mathrm{S}_{4}^{\mathrm{sm}}+\mathrm{S}_{2}^{\mathrm{a}}+$ sex chromosomes.
\end{abstract}

Key words Trachypithecus obscurus, Karyotype, Chromosome, Polymorpic NORs.

Langurs belong to a large group of old world monkeys called the colobines (subfamily Colobinae) and are distributed in tropical Asia. The colobines are unique among primates in that they are predominantly leaf eaters and exhibit foregut fermentation (Chivers and Hladik 1980). It is generally agreed that the colobines consist of 2 groups, or clades; the African colobus monkeys and

*Corresponding author, e-mail: tanomtong@hotmail.com

DOI: $10.1508 /$ cytologia. 77.131 
the Asian langur and leaf monkeys (Oates et al. 1994, Morales et al. 1999). Asian colobines have been divided into 5 or 6 species groups depending on the author (Napier and Napier 1985, Oates et al. 1994, Groves 2001, Brandon-Jones et al. 2004). According to the most recent classifications (Groves 2001, Brandon-Jones et al. 2004), the Asian colobines consist of 5 species groups. These include langurs (Semnopithecus), leaf monkeys (Trachypithecus), surili (Presbytis), snub-nosed monkeys (Rhinopithecus and Pygathrix), proboscis monkey (Nasalis), and pig-tailed monkey (Simias). In south Asia, colobines are represented by 2 genera: Semnopithecus and Trachypithecus (Karanth 2010).

There are 13 families, 60 genera and about 232 species of animals in the order Primate (Wilson and Cole 2000); however, only 3 families, 5 genera and 13 species were found in Thailand. The langur species consists of 2 genera and 4 species in Thailand, namely; banded langur (Presbytis femoralis Raffles, 1821), silvered leaf monkey (Trachypithecus cristatus Raffles, 1821), dusky leaf monkey (T. obscurus Ried, 1837), and Phryre's leaf monkey (T. phayrei Blyth, 1847) (Lekagul and McNeely 1988). The common characteristics of the T. obscurus are white spectacles on a dark face. The body is dark gray to blackish brown, contrasting with lighter hind legs and tail. There is a white patch of skin on the upper and lower lips. Young are bright golden-yellow (Parr 2003) (Fig. 1).

Inccordance with to the cytogenetic studies of T. obscurus by Chiarelli (1963), Hsu and Benirschke (1971) and Sangpakdee et al. (2008), in this study, we confirm and compare our findings with previous reports. In addition, this is the first report about standardized karyotypes and idiograms by CBG-banding and high-resolution techniques, and polymorphic NORs of T. obscurus. Thus, it is important to conduct this study, as it's findings will represent basic knowledge and can be applied to accommodate further research.

Materials and methods

Blood samples of the T. obscurus ( 2 males and 1 female) were collected from Songkla Zoo, Thailand and then applied to cytogenetic studies by lymphocyte culture of whole blood samples. The culture cells were treated with a colchicine-hypotonic-fixation-air-drying technique followed by conventional staining, GTG-, CBG-banding and high-resolution techniques (Rooney 2001, Campiranon 2003). For 20 cells of each individual chromosome, checks, length measurements, karotyping and idiograming were accomplished by using a light microscope as previously described (Chaiyasut 1989).

\section{Results and discussion}

The cytogenetic study of T. obscurus using lymphocyte culture demonstrated that the chromosome number is $2 n$ (diploid) $=44$ and the fundamental number ( $\mathrm{NF}$, number of chromosome arms) for both sexes is 88 (Fig. 2). These results agree with Chiarelli (1963), Hsu and Benirschke (1971) and Sangpakdee et al. (2008). According to the chromosome characteristics of others in the subfamily Colobinae in Thailand, T. cristatus $(2 n=44, \mathrm{NF}=88$; Hsu and Benirschke 1970, Ponsà et al. 1983, Bigoni et al. 1997) and T. phayrei $(2 n=44, \mathrm{NF}=88$; Nie et al. 1998), all of them have the same of $2 n=44$ and most have $\mathrm{NF}=88$.

The autosomes of T. obscurus consist of 6 large metacentric, 12 large submetacentric, 2 large acrocentric, 4 medium metacentric, 10 medium submetacentric, 2 small metacentric, 4 small submetacentric, and 2 small acrocentric chromosomes. This differs from the reports of Chiarelli (1963) and Hsu and Benirschke (1971) which demonstrated that the autosomes of T. obscurus are 40 metacentric and submetacentric, and 2 acrocentric chromosomes. The $\mathrm{X}$ chromosome of T. obscurus is a large submetacentric chromosome and the $\mathrm{Y}$ chromosome is a small submetacentric chromosome, 


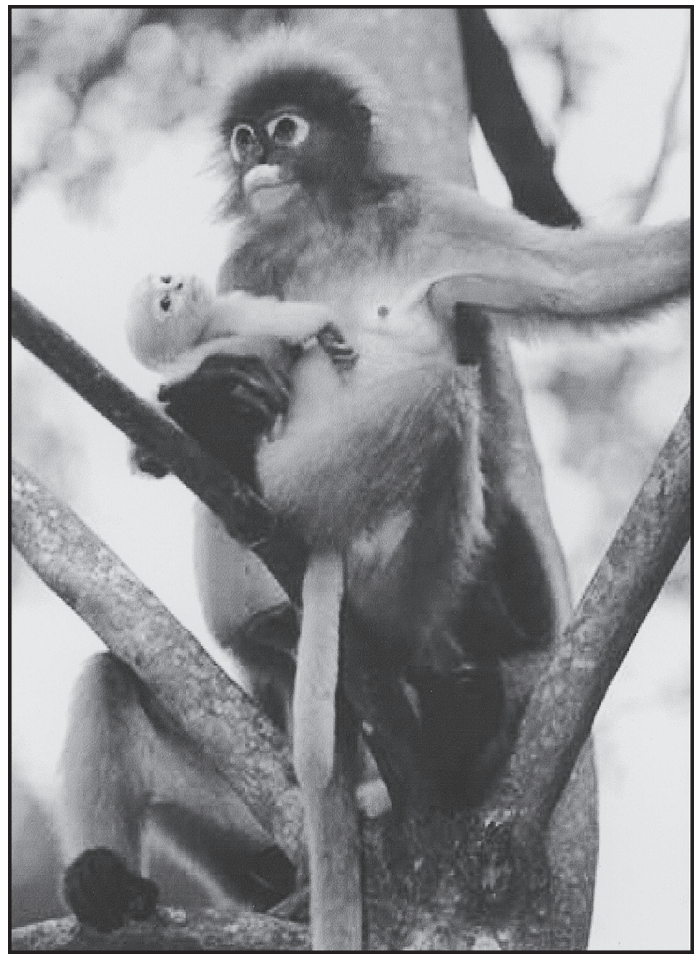

Fig. 1. General characteristics of the dusky leaf monkey, Trachypithecus obscures (Primate, Colobinae).
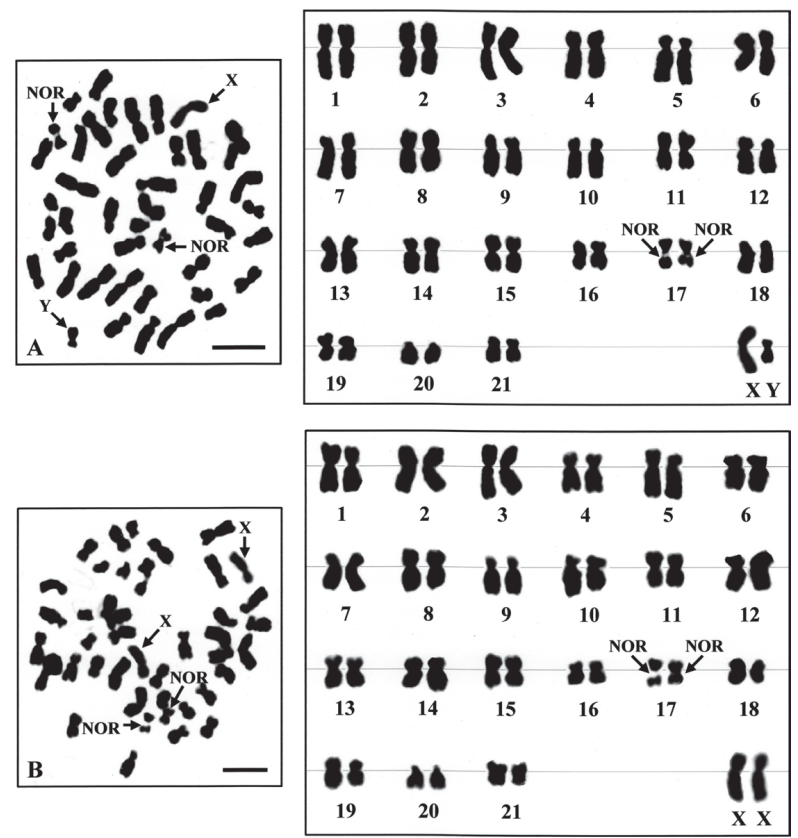

Fig. 2. Metaphase chromosome plates and karyotypes of male (A) and female (B) dusky leaf monkey (Trachypithecus obscures), $2 n=44$ by conventional staining technique. New discovery of polymorphic nature NORs of female in size differences of chromosome pair 17 , scale bars $=10 \mu \mathrm{m}$. 

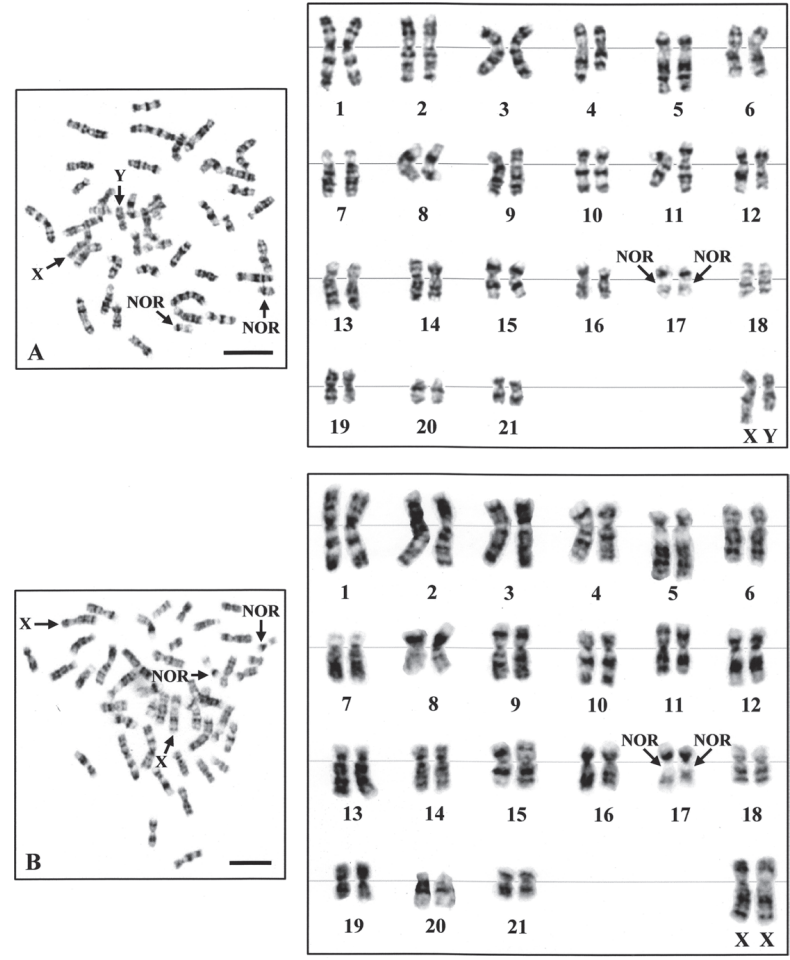

Fig. 3. Metaphase chromosome plates and karyotypes of male (A) and female (B) dusky leaf monkey (Trachypithecus obscures), $2 n=44$ by GTG-banding technique. New discovery of polymorphic nature NORs of female in size differences of chromosome pair 17 , scale bars $=10 \mu \mathrm{m}$.
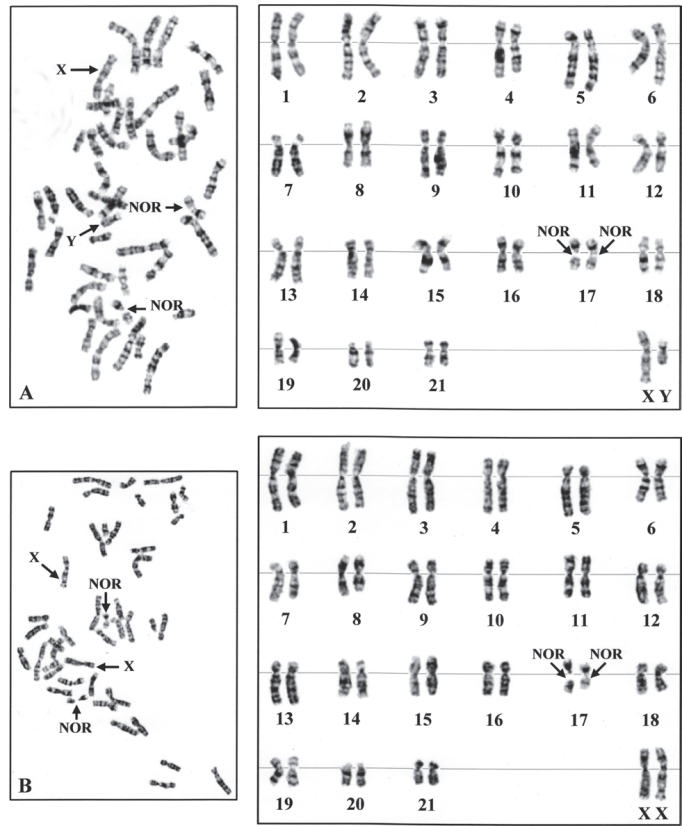

Fig. 4. Metaphase chromosome plates and karyotypes of male (A) and female (B) dusky leaf monkey (Trachypithecus obscures), $2 n=44$ by high-resolution technique. New discovery of polymorphic nature NORs of female in size differences of chromosome pair 17 , scale bars $=10 \mu \mathrm{m}$. 

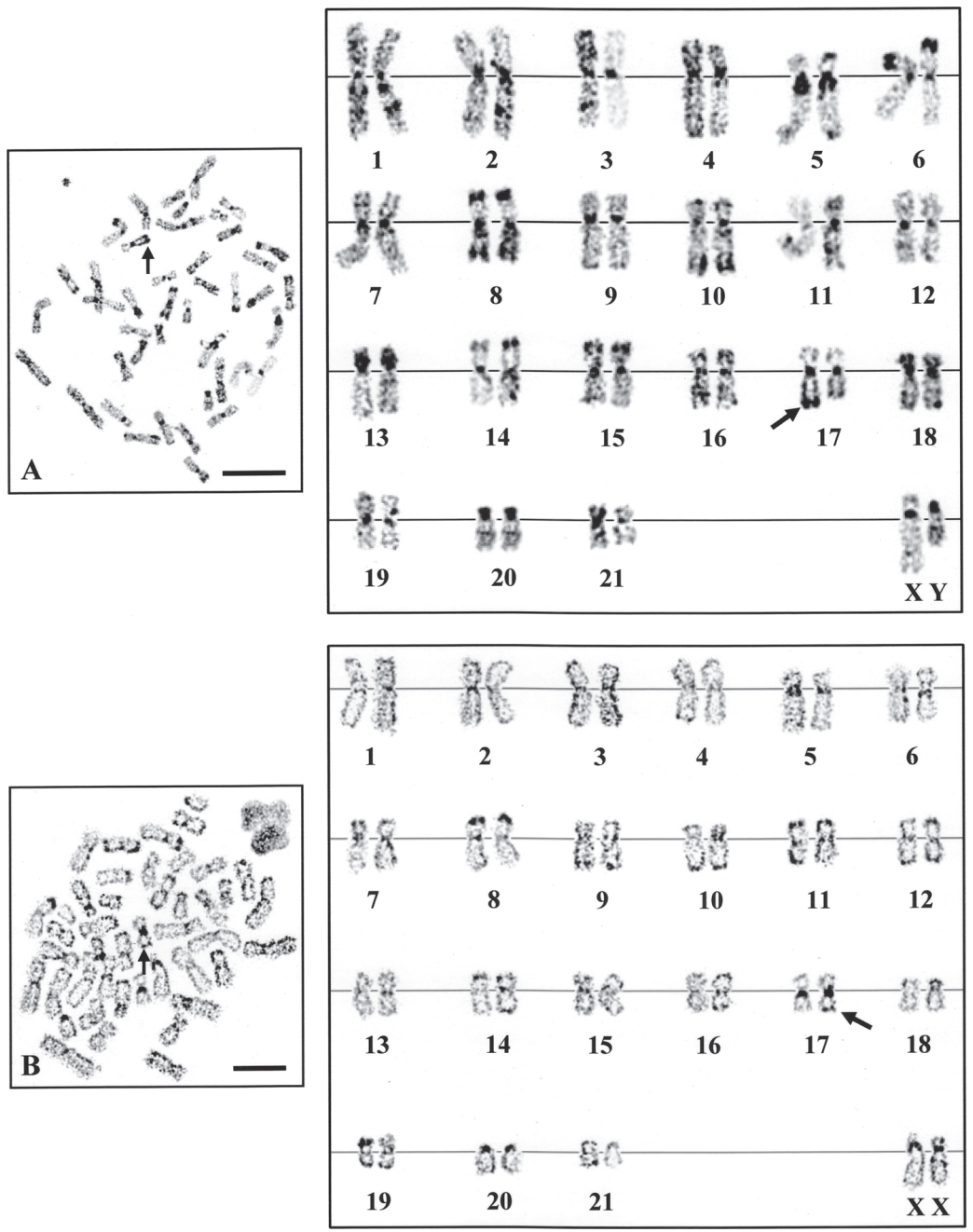

Fig. 5. Metaphase chromosome plates and karyotypes of male (A) and female (B) dusky leaf monkey (Trachypithecus obscures), $2 n=44$ by CBG-banding technique. Arrows indicate polymorphic nature NORs in C-positive (dark bands) of telomeres of long arms of chromosome pair 17, scale bars= $10 \mu \mathrm{m}$.

is similar to the reports of Hsu and Benirschke (1971). This feature is different to the report of Chiarelli (1963) that revealed the T. obscurus have a metacentric X chromosome and an acrocentric $\mathrm{Y}$ chromosome. In contrast with the subfamily Colobinae in Thailand, the $\mathrm{X}$ and $\mathrm{Y}$ chromosomes of T. cristatus and T. phayrei are submetacentric chromosomes (Hsu and Benirschke 1970, Ponsà et al. 1983, Bigoni et al. 1997, Nie et al. 1998).

In addition, a pair of long arms near the centromeres of chromosome 17 showed clearly observable NORs. This is the first report on nature polymorphism in NORs in T. obscurus. The results showed a heteromorphism in 1 female with a difference in the size of NORs of chromosome 17, while the 2 males, the results showed an equal size of both chromosome $17 \mathrm{~s}$ with a homomorphism that is consistent to the report of Warburton et al. (1975) that found a difference in the size of NORs (polymorphism) in the white-handed gibbon (Hylobates lar), while Tanomtong et al. (2010) found the same polymorphic NORs in dark-hand gibbons (H. agilis). Nucleolar organizer regions are composed of high amounts of rDNA, protein and RNA. Ribosomal DNA functions on 28S and 


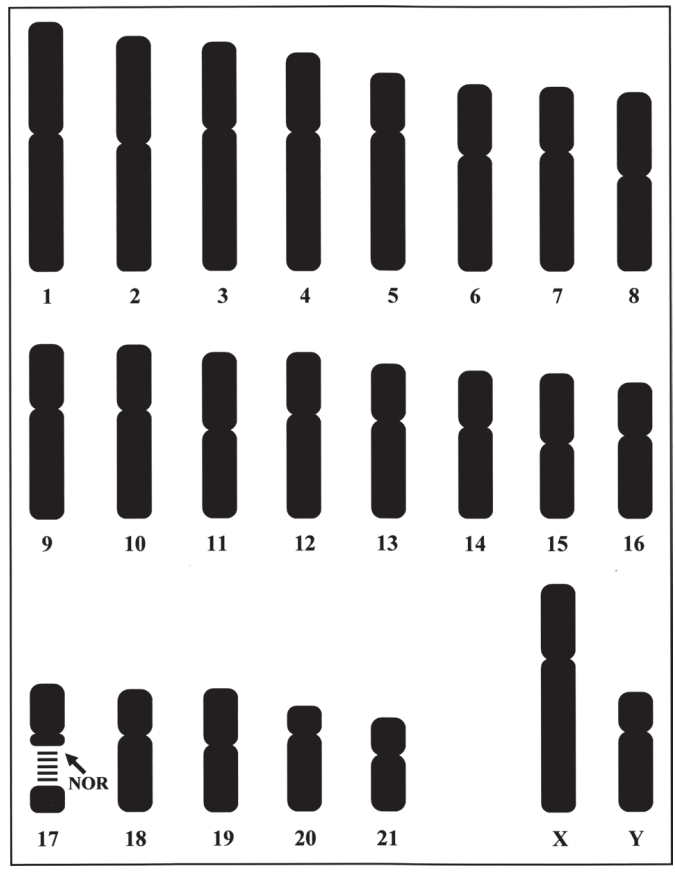

Fig. 6. Idiogram of dusky leaf monkey (Trachypithecus obscures), $2 n=44$ by conventional staining technique, showing nucleolar organizer region, NOR (arrow).

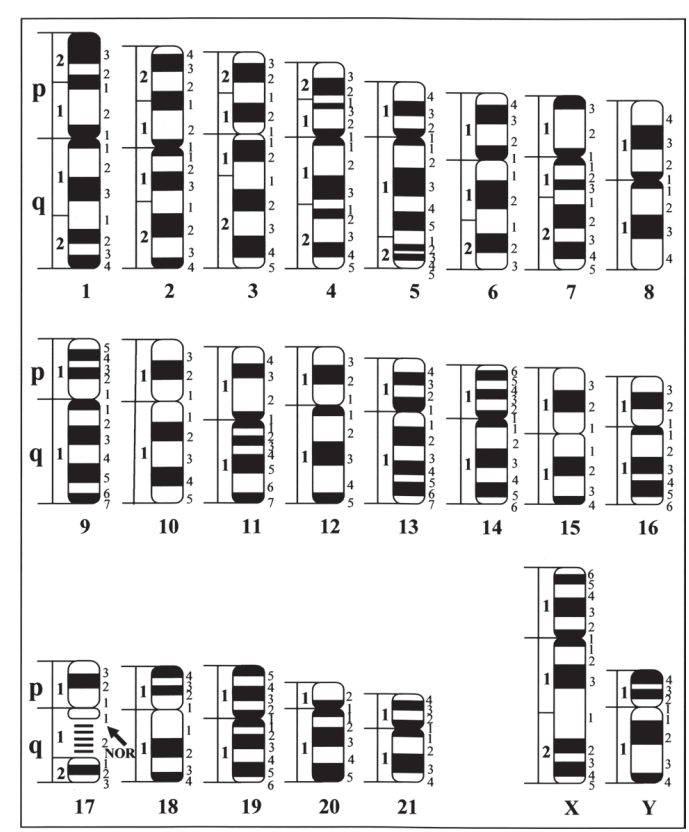

Fig. 7. Idiogram of dusky leaf monkey (Trachypithecus obscures), $2 n=44$ by GTG-banding technique, showing nucleolar organizer region, NOR (arrow).

18S rRNA synthesis in mammals. The rDNA, repetitive DNA, function in rRNA synthesis, so the increase or decrease in the size of NORs in T. obscurus might influence the genes in protein synthesis (Campiranon 2003). Warburton et al. (1975) found a polymorphism NORs in macaques (genus Macaca). However, Tantravahi et al. (1976) and Miller et al. (1977) reported that there are NORs on human chromosomes 13, 14, 15, 21, 22 and also found that human satellite chromosomes have polymorphism by NOR-banding techniques.

The GTG-banding technique revealed that the number of GTG-bands on 1 set of haploid chromosomes $(n)$, which includes autosomes, and the $\mathrm{X}$ and $\mathrm{Y}$ chromosome, is 235 bands for the T. obscurus. The number of bands in 1 set of prometaphase haploid chromosomes from the high-resolution technique is 314 (Figs. 7 and 8). This can be compared with the study in human and apes by Yunis (1982), which reported that the chromosome band number from the high-resolution technique of prometaphase chromosomes is over 1,000 bands per haploid set. In this study, the chromosome scoring is done only with clearly visible bands, except for variable bands due to the small number of scored bands.

The CBG-banding technique demonstrated dark bands (C-positive) on the centromeres of all chromosomes (autosome pairs 1-21 and X chromosome) but light bands or C-negative on the Y chromosome. The CBG-banding can provide a dark region on chromosomes which represents the constitutive heterochromatin of chromosomes that can be found at all centromeres and some telomeres of normal chromosomes. CBG-banding is the accepted technique for the study of sex chromosomes, especially for the identification of the $\mathrm{Y}$ chromosome because of its individual characteristics, which normally fail to provide a dark region on the centromere (Campiranon, 2003).

The CBG-banding technique showed interstitial dark bands on the chromosomes. Results gained from this technique demonstrated that chromosome pair 1 had 2 interstitial large dark bands 


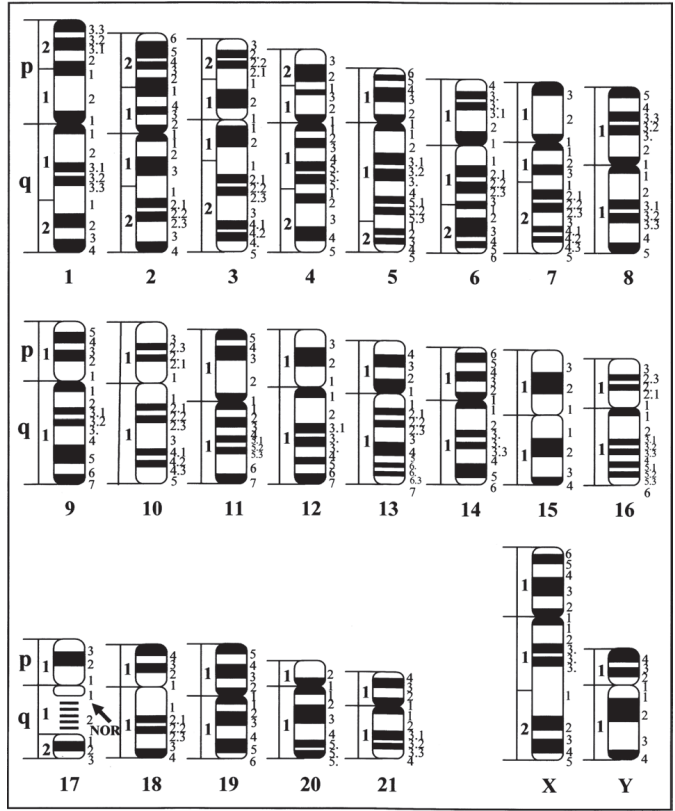

Fig. 8. Idiogram of dusky leaf monkey (Trachypithecus obscures), $2 n=44$ by high-resolution technique, showing nucleolar organizer region, NOR (arrow).

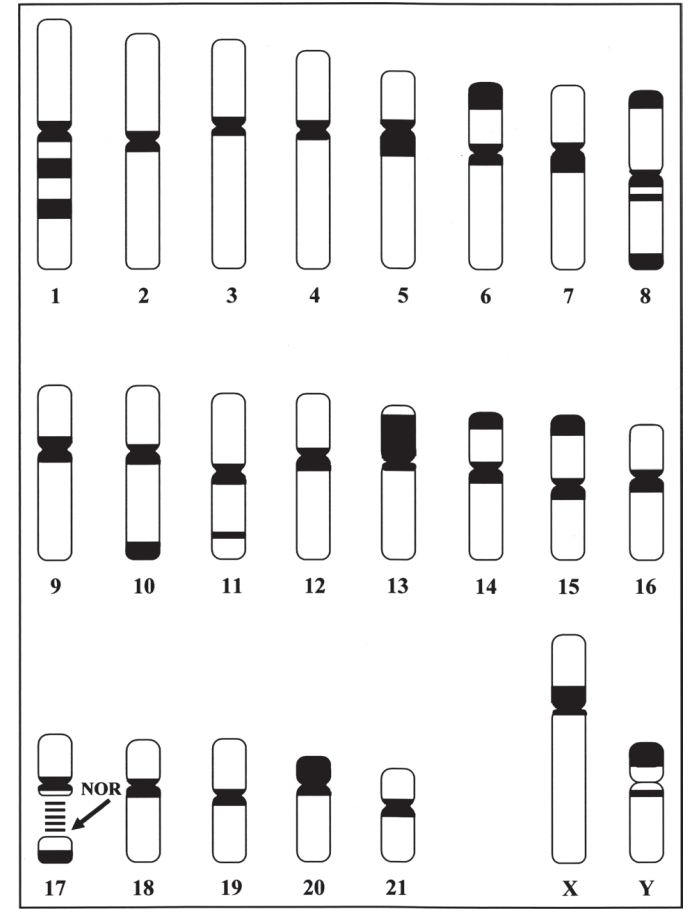

Fig. 9. Idiogram of dusky leaf monkey (Trachypithecus obscures), $2 n=44$ by CBG-banding technique, showing C-positives in dark bands and nucleolar organizer region, NOR (arrow).

on the long arm; chromosome pairs 6,14 and 15 had a large dark band on the telomeres of the short arms; chromosome pair 8 had large dark bands on the telomeres of both long and short arms and also showed a small dark band on the long arm; chromosome pair 10 had an interstitial large dark band on the telomere of the long arm; chromosome pair 11 showed an interstitial small dark band on the long arm; Y chromosome had an interstitial large dark band on the telomere while a small one exists at the long arm. For chromosome pair 17, the presence of polymorphic NORs, stained with this technique, revealed a dark band on the telomere of only one long arm (heteromorphism). In addition, chromosome pairs 13 and 20 showed very large dark bands on the short arms (Fig. 9). Although normal chromosomes have dark bands at the centromere (constitutive heterochromatin), the existence of an interstitial dark band on a chromosome or at a telomere refers to the fact that centric fusion or tandem fusion during the chromosomal evolutionary process of $T$. obscurus are the factors which played important role in the combination between 2 arm chromosome (telocentric chromosome) and the decrement of chromosome number but the constant of fundamental number. The combination described above, 1 bi-arm pattern, does not affect the location of centromeres; it means that staining with CBG-banding technique still provides interstitial dark bands on chromosomes or telomeres.

The chromosomes of mitotic metaphase cells and the karyotypes of T. obscurus examined by conventional, GTG-, CBG-banding and high-resolution techniques are shown in Figs. 2-5. The chromosomes length in centimeters of 20 cells (male and female) in mitotic metaphase was measured. The mean length of the short arm chromosome (Ls), length of the long arm chromosome (L1), total length of the arm chromosome (LT), relative length (RL), centromeric index (CI), and 
Table 1. Mean of length short arm chromosome (Ls), length of long arm chromosome (L1), length of total arm chromosome (LT), relative length (RL), centromeric index (CI) and standard deviation (SD) of RL, CI from metaphase chromosomes in 20 cells of the dusky leaf monkey (Trachypithecus obscures) $2 n=44$.

\begin{tabular}{cccccccc}
\hline \hline $\begin{array}{c}\text { Chromosome } \\
\text { pairs }\end{array}$ & Ls & L1 & LT & RL \pm SD & CI \pm SD & $\begin{array}{c}\text { Chromosome } \\
\text { size }\end{array}$ & $\begin{array}{c}\text { Chromosome } \\
\text { type }\end{array}$ \\
\hline 1 & 0.619 & 0.759 & 1.378 & $0.549 \pm 0.021$ & $0.034 \pm 0.002$ & Large & Metacentric \\
2 & 0.595 & 0.701 & 1.296 & $0.541 \pm 0.030$ & $0.032 \pm 0.002$ & Large & Metacentric \\
3 & 0.480 & 0.783 & 1.263 & $0.620 \pm 0.067$ & $0.031 \pm 0.003$ & Large & Submetacentric \\
4 & 0.433 & 0.768 & 1.200 & $0.640 \pm 0.071$ & $0.030 \pm 0.002$ & Large & Submetacentric \\
5 & 0.322 & 0.767 & 1.089 & $0.705 \pm 0.069$ & $0.027 \pm 0.003$ & Large & Acrocentric \\
6 & 0.392 & 0.637 & 1.028 & $0.620 \pm 0.061$ & $0.025 \pm 0.002$ & Large & Submetacentric \\
7 & 0.356 & 0.660 & 1.016 & $0.648 \pm 0.049$ & $0.025 \pm 0.002$ & Large & Submetacentric \\
8 & 0.460 & 0.522 & 0.982 & $0.530 \pm 0.023$ & $0.024 \pm 0.002$ & Large & Metacentric \\
9 & 0.353 & 0.609 & 0.961 & $0.633 \pm 0.049$ & $0.024 \pm 0.001$ & Large & Submetacentric \\
10 & 0.361 & 0.597 & 0.958 & $0.629 \pm 0.043$ & $0.024 \pm 0.002$ & Large & Submetacentric \\
11 & 0.425 & 0.484 & 0.909 & $0.531 \pm 0.019$ & $0.022 \pm 0.002$ & Medium & Metacentric \\
12 & 0.339 & 0.571 & 0.909 & $0.627 \pm 0.031$ & $0.022 \pm 0.002$ & Medium & Submetacentric \\
13 & 0.312 & 0.535 & 0.847 & $0.626 \pm 0.058$ & $0.021 \pm 0.002$ & Medium & Submetacentric \\
14 & 0.311 & 0.502 & 0.813 & $0.618 \pm 0.036$ & $0.020 \pm 0.001$ & Medium & Submetacentric \\
15 & 0.384 & 0.410 & 0.794 & $0.516 \pm 0.020$ & $0.020 \pm 0.003$ & Medium & Metacentric \\
16 & 0.291 & 0.456 & 0.747 & $0.610 \pm 0.052$ & $0.018 \pm 0.002$ & Medium & Submetacentric \\
$17 *$ & 0.275 & 0.436 & 0.711 & $0.614 \pm 0.031$ & $0.018 \pm 0.002$ & Medium & Submetacentric \\
18 & 0.260 & 0.422 & 0.682 & $0.619 \pm 0.036$ & $0.017 \pm 0.001$ & Small & Submetacentric \\
19 & 0.310 & 0.371 & 0.681 & $0.546 \pm 0.033$ & $0.017 \pm 0.002$ & Small & Metacentric \\
20 & 0.150 & 0.427 & 0.577 & $0.738 \pm 0.041$ & $0.014 \pm 0.001$ & Small & Acrocentric \\
21 & 0.201 & 0.314 & 0.515 & $0.610 \pm 0.037$ & $0.013 \pm 0.001$ & Small & Submetacentric \\
$\mathrm{X}$ & 0.411 & 0.850 & 1.261 & $0.670 \pm 0.075$ & $0.031 \pm 0.006$ & Large & Submetacentric \\
$\mathrm{Y}$ & 0.215 & 0.444 & 0.659 & $0.676 \pm 0.047$ & $0.016 \pm 0.004$ & Small & Submetacentric \\
\hline & & & & & & &
\end{tabular}

Remarks: $*=$ satellite chromosome (NORs).

standard deviation of RL, CI, size and type of chromosome are presented in Table 1. The idiogram of $T$. obscurus shows the gradually decreasing length of the chromosomes.

The T. obscurus demonstrated that the chromosome markers are the autosome pair 1 and pair 21 which are the largest metacentric chromosome and the smallest submetacentric chromosome, respectively. The important karyotype feature of T. obscurus is the asymmetrical karyotypes, which were found in all 3 types of chromosomes (metacentric, submetacentric and acrocentric chromosomes). The largest chromosome is 3 times larger than the smallest chromosome. Figures 6-9 show the idiograms of the T. obscurus from conventional, GTG-, CBG-banding and high-resolution techniques. The karyotype formula for T. obscurus could be deduced as: $2 n$ (diploid) $44=\mathrm{L}_{6}^{\mathrm{m}}+\mathrm{L}_{12}^{\mathrm{sm}}+\mathrm{L}_{2}^{\mathrm{a}}+$ $\mathrm{M}_{4}^{\mathrm{m}}+\mathrm{M}_{10}^{\mathrm{sm}}+\mathrm{S}_{2}^{\mathrm{m}}+\mathrm{S}_{4}^{\mathrm{sm}}+\mathrm{S}_{2}^{\mathrm{a}}+$ sex chromosomes.

\section{Acknowledgements}

This work was supported by the Applied Taxonomic Research Center (ATRC), Khon Kaen University grant ATRC-R5304 and we also thank the director of Nakhon Ratchasima Zoo for valuable help. Thanks are also expressed intended to the staff of this zoo for their good cooperation. We wish to acknowledge the support of the Khon Kaen University Publication Clinic, Research and Technology Transfer Affairs, Khon Kaen University, for their assistance.

\section{References}

Bigoni, F., Koehler, U., Stanyon, R., Ishida, T. and Wienberg, J. 1997. Fluorescence in situ hybridization establishes ho- 
mology between human and silvered leaf monkey chromosomes, reveals reciprocal translocations between chromosomes homologous to human $\mathrm{Y} / 5,1 / 9$ and 6/16 and delineates an $\mathrm{X}_{1} \mathrm{X}_{2} \mathrm{Y}_{1} \mathrm{Y}_{2} / \mathrm{X}_{1} \mathrm{X}_{1} \mathrm{X}_{2} \mathrm{X}_{2}$ sex-chromosome system. Am. J. Phys. Anthropol. 23: 315-327.

Brandon-Jones, D., Eudey, A. A., Geissmann, T., Groves, C. P., Melnick, D. J. and Morales, J. C. 2004. Asian primate classification. Int. J. Primatol. 25: 97-162.

Campiranon, A. 2003. Cytogenetics. 2nd ed. Department of Genetics, Faculty of Science, Kasetsart University, Bangkok.

Chaiyasut, K. 1989. Cytogenetics and cytotaxonomy of the family Zephyranthes. Department of Botany, Faculty of Science, Chulalongkorn University, Bangkok.

Chiarelli, B. 1963. Comparative morphometric analysis of primate chromosomes. III. The chromosome of the genera Hylobates, Colobus and Presbytis. Caryologia 16: 637-648.

Chivers, D. J. and Hladik, C. M. 1980. Morphology of the gastrointestinal tract in primates: comparisons with other mammals in relation to diet. J. Morphol. 166: 377-386.

Groves, C. P. 2001. Primate taxonomy. Smithsonian Institute Press, Washington.

Hsu, T. C. and Benirschke, K. 1970. Presbytis cristatus (silvered leaf-monkey) $2 n=44$. In: An atlas of mammalian chromosomes. Hsu, T. C. and Benirschke, K. (eds.) Folio 199. Springer-Verlag, New York. pp. 214-216.

- and - 1971. Presbytis obscurus (dusky langur) $2 n=44$. In: An atlas of mammalian chromosomes. Hsu, T.C. and Benirschke, K. (eds.) Folio 249. Springer-Verlag, New York. pp. 143-145.

Karanth, K. P. 2010. Molecular systematics and conservation of the langurs and leaf monkeys of South Asia. J. Genet. 89: 393-399.

Lekagul, B. and McNeely, J. A. 1988. Mammals of Thailand. 2nd ed. Sahakarn Bhaet, Bangkok.

Miller, D. A. 1977. Evolution of primate chromosomes. Science 198: 1116-1124.

Morales, C. J., Disotell, T. R. and Melnick, D. J. 1999. Molecular phylogenetic studies of nonhuman primates. In: The nonhuman primates. Dolhinow, P. and Fuentes, A. (eds.). Mayfield Publishing Company, Mountain View. pp. 18-38.

Napier, J. R. and Napier, P. H. 1985. The natural history of the primates. MIT Press, Cambridge.

Nie, W., Liu, R., Chen, Y., Wang, J. and Yang, F. 1998. Mapping chromosomal homologies between humans and two langurs (Semnopithecus francoisi and S. phayrei) by chromosome painting. Chromosomal Research 6: 447-453.

Oates, J. F., Davies, A. G. and Delson, E. 1994. The diversity of living colobines. In: Colobine monkeys: their ecology, behaviour and evolution. Davies, A. G. and Oates, J. F. (eds.). Cambridge University Press, Cambridge. pp. 45-73.

Parr, J. W. K. 2003. Large mammals of Thailand. Sarakadee Press, Bangkok.

Ponsà, M., De Boer, L. E. M. and Egozcue, J. 1983. Banding pattern of the chromosomes of Presbytis cristatus pyrrhus and P. obscurus. Am. J. Primatol. 4: 165-169.

Rooney, D. E. 2001. Human cytogenetics: constitutional analysis. Oxford University Press, Oxford.

Sangpakdee, W., Tanomtong, A., Monthatong, M., Pinthong, K., Gomontean, B. and Nie, W. 2008. The homology and relationship of human (Homo sapiens) chromosomes 1, 19 and dusky langur (Trachypithacus obscurus) chromosomes 6, 8 demonstrated with chromosome painting. Cytologia 73: 349-355.

Tanomtong, A., Khunsook, S., Supanuam, P. and Kaewsri, S. 2010. A discovery of polymorphism of nucleolar organizer regions (NORs) and whole-arm translocation (WAT) between chromosome 8 and 9 of lowland agile gibbon (Hylobates agilis unko) in Thailand. Cytologia 75: 15-21.

Tantravahi, R., Miller, D. A., Dev, V. G. and Miller, O. J. 1976. Detection of nucleolus organizer region in chromosomes of human, chimpanzee, gorilla, orangutan and gibbon. Chromosoma 56: 15-27.

Warburton, D., Handerson, A. S. and Atwood, K. C. 1975. Localization of rDNA and Giemsa-banded chromosome complement of white-handed gibbon, Hylobates lar. Chromosoma 51: 35-40.

Wilson, D. E. and Cole, F. R. 2000. Common names of mammals of the world. Smithsonian Institution Press, Washington.

Yunis, J. J. and Prakash, O. 1982. The origin of man: A chromosome pictorial legacy. Science 215: 1525-1530. 